JASC 12-1-9

\title{
Motor noise removal for determining gait events over treadmill walking using wavelet filter
}

\author{
Ho Jun Yeom ${ }^{\dagger}$, Brian P. Selgrade ${ }^{*}$, Young Hui Chang ${ }^{*}$, Jung Lae Kim ${ }^{\dagger \dagger}$ \\ ${ }^{\dagger}$ Dept. of Medical Engineering, Eulji University, Seongnam-si, Gyounggi-do, Republic of Korea \\ *Dept. of Applied Physiology, Georgia Institute of Technology, Atlanta, Georgia , USA
}

\begin{abstract}
The conventional method for filtering force plate data, low-pass filtering, does not always give accurate results when applied to force data from a custom-made, instrumented treadmill. Therefore, this study compares low-pass filtered data to the same data passed through a wavelet filter. We collected data with the treadmill running. However these include motor noise with ground reaction force at two force plates. We found that he proposed wavelet method eliminated motor noise to result in more accurate force plate data than the conventional low-pass filter, particularly at high speed motor operation. In this study we suggested the convolution wavelet (CNW) which was compared to that of a low-pass filter. The CNW showed better performance as compared to band-pass filtering particularly for low signal-to-noise ratios, and a lower computational load.
\end{abstract}

Key words : Wavelet, Treadmill, Gait, Motor noise, Ground reaction force

\section{INTRODUCTION}

The standard method to filter data acquired from force plates is a low pass filter, which is used in many gait studies[1][2]. This is sufficient for over-ground studies, but, in the case of a custom-made, instrumented treadmill moving at high speeds, the treadmill motors produce additional noise that is not always removed by a low-pass filter. Center of pressure data is calculated from ground reaction force(GRF), and the center of pressure is used in inverse dynamics to find the moments created by the GRF about the leg joints. Therefore, accurate GRF data is critical to the accuracy of inverse dynamics calculations.

\footnotetext{
Manuscript received Jan. 26,2012; revisedFeb.23,2012

Corresponding Author:<jlkim@eulji.ac.kr> Tel:+82-31-740-7390,EuljiUniv.

*Dept. of Medical Engineering, Eulji Univ..Korea
}

Thus, noisy GRF data can be problematic in gait studies. Therefore, we seek to use a wavelet filter to remove the noise due to the treadmill, giving cleaner, moreaccurate GRF data. We hypothesize that the wavelet method will eliminate noise to result in more accurate force plate data than the low-pass filter, particularly at higher treadmill speeds.

\section{METHODS}

We obtained center of pressure data using a calibration stick with a marker at one end. With the calibration stick held close to vertical, the end with the marker was pressed against the moving treadmill track. The marker was to verify center of pressure data from the force plate using marker data given by a6-camera Vicon system. Marker position from camera data was compared with filtered force plate data using linear regression. To validate force measurements, separate trials were taken in which the treadmill was loaded with a calibration pole 
containing a force transducer. force transducer. Data from the transducer was compared with force data from the force plates. For both the calibration pole and calibration stick, we gathered data at $1 \mathrm{~m} / \mathrm{s}$ and $2 \mathrm{~m} / \mathrm{s}$ treadmill Data from the transducer was compared with force data from the force plates. For both the calibration pole and calibration stick, we gathered data at $1 \mathrm{~m} / \mathrm{sand} 2 \mathrm{~m} / \mathrm{s}$ treadmill speeds. Force data was processed in Matlab and filtered with two methods. Force data was first filtered with a standard method-a low-pass, four thorder, Butterworth filter with a threshold of $20 \mathrm{~Hz}$-to filter the raw force data.

The wavelet trans form is a convolution of the wavelet function $\psi(\mathrm{t})$ with the signal $\mathrm{x}(\mathrm{t})$. Orthonormal dyadic discrete wavelets are associated with scaling functions $\varphi$ (t). The scaling function can be convolved with the signal to produce approximation coefficients $\mathrm{S}$. The discrete wavelet transform(DWT) can be written as:

$$
T_{m, n}=\int_{-\infty}^{\infty} x(t) \psi_{m, n}(t) d t
$$

In practice our discrete input signal $\mathrm{S} 0, \mathrm{n}$ is of finite length $\mathrm{N}$, which is an integer power of $2: \mathrm{N}=2 \mathrm{M}$. Thus the range of scales that can be investigated is $0<\mathrm{m}<\mathrm{M}$ [5]. A discrete approximation of the signal can be shown as

$x_{0}(t)=x_{M}(t)+\sum_{m=1}^{M} d_{m}(t)$

where the mean signal approximation at scale $M$ is

$$
x_{M}(t)=S_{M, n} \phi_{M, n}(t)
$$

and the detail signal approximation corresponding to scale $\mathrm{m}$ is defined for a finite length signal as

$$
d_{m}(t)=\sum_{n=0}^{2^{M-m}-1} T_{m, n} \psi_{m, n}(t)
$$

The convolution wavelet method (CNW) method can reduce the computational complexity for real-time signal processing [6][7][8].

$$
C N W(t)=\psi(t)_{k=3} * \phi(t)_{k=3}
$$

(a) Scaling function

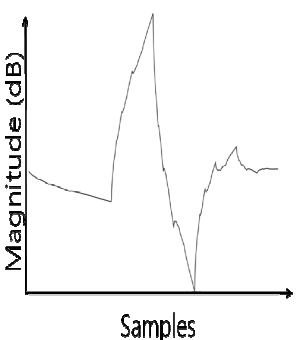

Fig. 1. The magnitude response of wavelet function and scaling function.

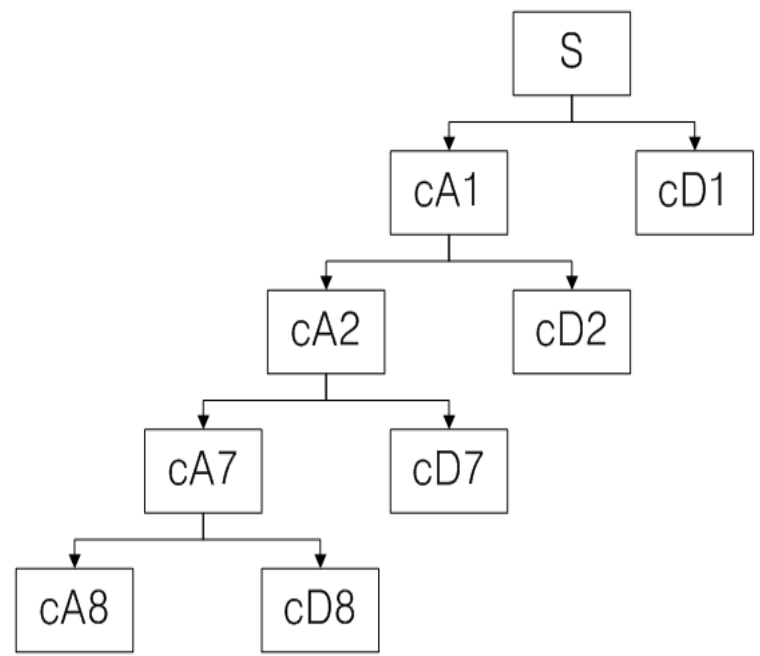

Fig 2. Diagram of 8 Level Decomposition

We selected and reconstructed cD8, cD7, cD6, cD5 which turned out to have the most of desired components through on 8-Levels Decomposition. Based on the experiment, we selected and reconstructed cD8, cD7 and cD6, cD5.

\section{RESULTS AND DISCUSSION}

The linear regression in Fig. 2 indicate that, while errors in center of pressure data after low-pass filtering may be acceptable at $1 \mathrm{~m} / \mathrm{s}$, these errors become too large when the treadmill runs at $2 \mathrm{~m} / \mathrm{s}$. This confirms that a different filter is necessary. We found that the result of $\mathrm{CNW}$ 
eliminated the noise while retaining all of the important components of the signal.
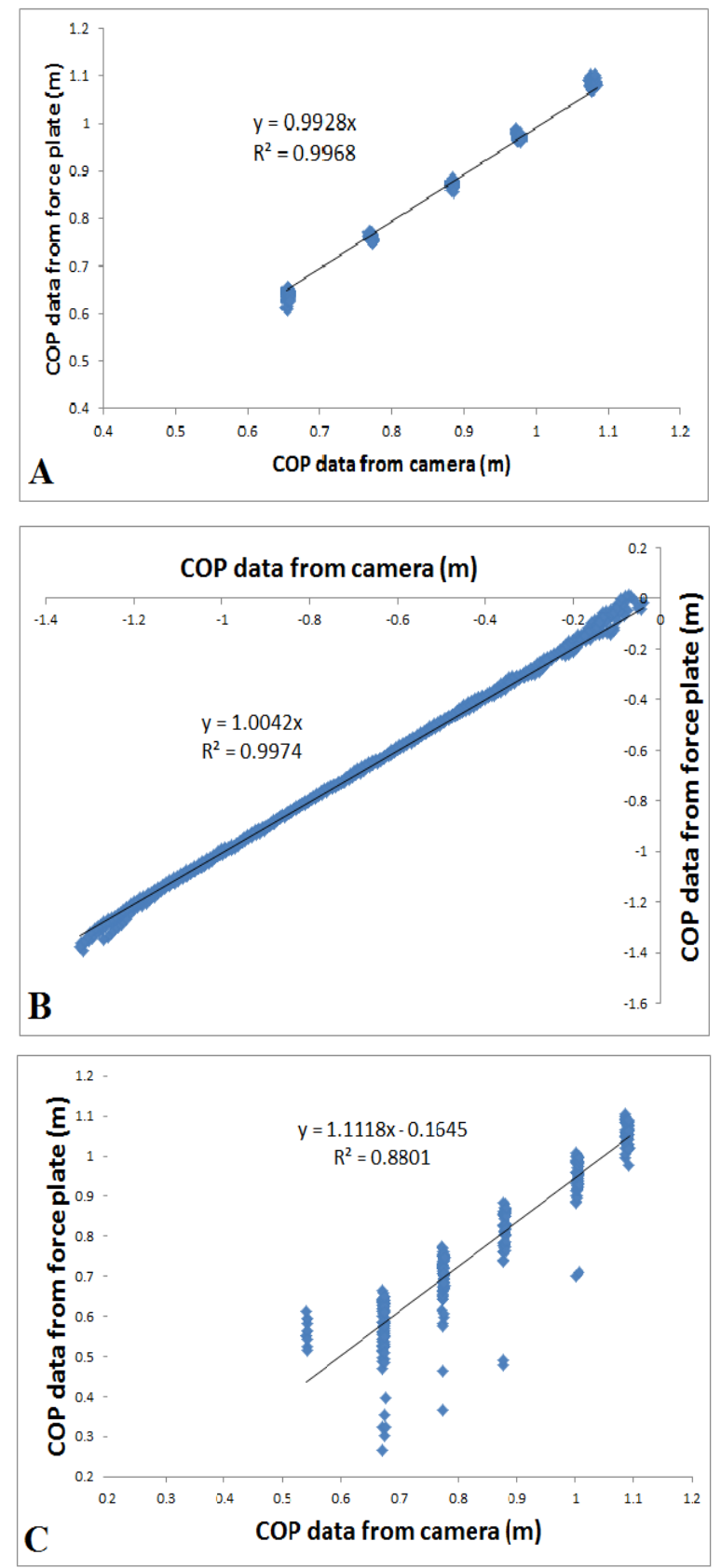

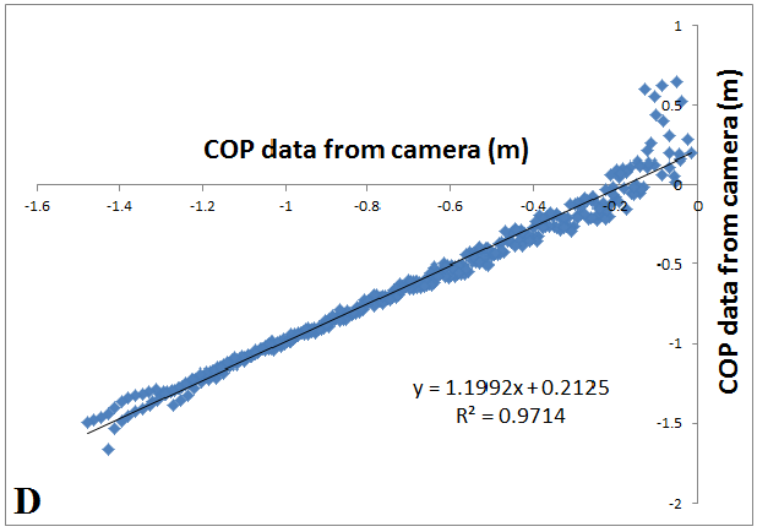

Fig. 3. The medial-lateral coordinate (A) and anterior-posterior coordinate (B) of COP at $1 \mathrm{~m} / \mathrm{s}$ and the medial-lateral coordinate $(\mathrm{C})$ and anterior-posterior coordinate (D) of COP at $2 \mathrm{~m} / \mathrm{s}$.

The instrumented treadmill can facilitate many types of biomechanical studies of human locomotionand measurevertical and horizontal ground-reaction forces during gait. Since wavelet filtering allows for more accurate force measurement at high speeds, it makes the instrumented treadmill a more powerful tool for gait analysis.

\section{ACKNOWLEDGMENT}

This work (Grants No. 00048056-1) was supported by Business for Cooperative R\&D between Industry, Academy, and Research Institute funded Korea Small and Medium Business Administration in 2011.

\section{REFERENCES}

[1] Yen, J., Auyang, A., Chang, Y., "Joint-level kinetic redundancy is exploited to control limb-level forces during human hopping". Exp Brain Res Vol.196, pp.439-51, 2009.

[2] Hobara, H., Inoue, K., Omuro, K., Muraoka, T., Kanosue, $\mathrm{K}$. "Determinant of leg stiffness during hopping is frequency-dependent". Eur J Appl Phys Vol.111, No. 9, pp. 2195-2201, 2011.

[3] Yoon, U. Noh, Y. Yoon, H. "Optimization methods for improving the performance of heart rate detection by a wearable ECG system during high-intensity exercise", Biomedical Engineering Letters, vol.2, pp.143-150, 2011.

[4] Mahmoodabadi S. Z., Ahmadian A., Abolhasani M. D. "Ecg Feature Extraction Using Daubechies Wavelets", 
Proceedings of the Fifth IASTED Visualization Imaging, and Image Processing, pp. 343-348, Sep. 2005

[5] Hojun Yeom,: "Compensation of Skin Surface Temperature Variation on the PPG for the U-Healthcare System", Journal of the Institute of Webcasting, Internet and Telecommunication, vol. 11 no. 6, December, 2011.

[6] Joong-Hee Lim, Inn-Ho Jee, "DWT, Dual Tree Discrete Wavelet Transform(DDWT), Quincunx, Non-separable Processing", Journal of the Institute of Webcasting, Internet and Telecommunication, vol. 12 no. 1, February 2012

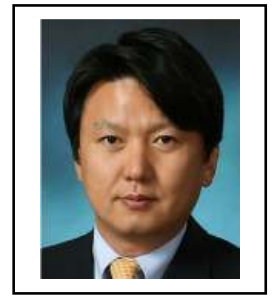

Ho Jun Yeom received his BS, MS and $\mathrm{Ph} . \mathrm{D}$ in Biomedical Engineering from Yonsei University. He has been an Assistant Professor of Medical Engineering at the Eulji University since 2008. His main research interests involve studying rehabilitation engineering and biomedical signal processing.

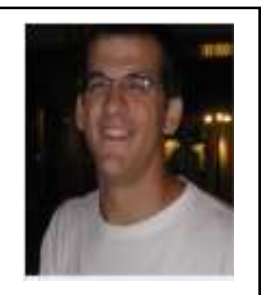

Brian Selgrade received his BS in biology from North Carolina State University and his MS in biomedical engineering from Duke University.

$\mathrm{He}$ has been a $\mathrm{PhD}$ student in the Neuromechanics Lab at Georgia Institute of Technology since 2010. His research interests include motor learning and the biomechanics of locomotion.

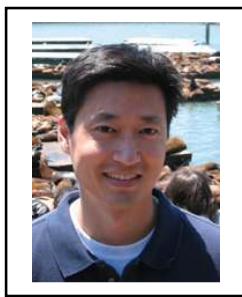

Young Hui Chang received his BS in Mechanical and Aerospace Engineering from Cornell University and his MS in Animal Physiology from the Cornell University College of Veterinary Medicine. In 2000 he completed his $\mathrm{PhD}$ in Integrative Biology at the University of California, Berkeley. He has been an Assistant Professor of Applied Physiology at the Georgia Institute of Technology since 2004 where directs the Comparative Neuromechanics Laboratory. His main research interests involve studying biomechanics and motor control of the legs during vertebrate locomotion. 